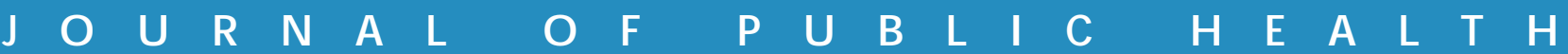

\title{
Diabetes como causa básica ou associada de morte no Estado de São Paulo, Brazil, 1992
}

\section{Diabetes as underlying or associated cause of death in the State of S. Paulo, 1992}

Laercio J. Franco, Cecília Mameri, Heloisa Pagliaro, Lúcia C. Iochida e Paulete G oldenberg Departamento de Medicina Preventiva da Universidade Federal de São Paulo. São Paulo, SP-Brasil (L.J.F., H.P., L.C.I.), Fundação Sistema Estadual de Análises de Dados. São Paulo, SP - Brasil (C.M.) 


\title{
Diabetes como causa básica ou associada de morte no Estado de São Paulo, Brazil, 1992
}

\author{
Diabetes as underlying or associated cause of death in the State \\ of S. Paulo, 1992
}

\author{
Laercio J. Franco, Cecília Mameri, Heloisa Pagliaro, Lúcia C. Iochida e Paulete G oldenberg \\ Departamento de Medicina Preventiva da Universidade Federal de São Paulo. São Paulo, SP-Brasil \\ (L.J.F., H.P., L.C.I.), Fundação Sistema Estadual de Análises de Dados. São Paulo, SP - Brasil (C.M.)
}

\begin{abstract}
Introdução As estatísticas de mortalidade, em geral, baseiam-se na análise das causas básicas de óbito. No caso do diabetes, sua importância é sempre subestimada, pois os diabéticos geralmente morrem devido às complicações crônicas da doença, sendo estas que figuram como a causa básica do óbito. Para atenuar esse problema, deveriam ser analisadas todas as causas mencionadas no atestado de óbito. Como contribuição ao problema foi analisada a freqüência das menções do diabetes nas declarações de óbito e as principais causas associadas.
\end{abstract}

Metodologia Os coeficientes específicos e a mortalidade proporcional por diabetes, como causa básica ou associada, foram calculados com base nas informações extraídas dos atestados de óbito, através do sistema ACME (Automated Classification of Medical Entities), para o Estado de São Paulo, em1992.

Resultados e Conclusões

\begin{abstract}
De um total de 202.141 óbitos, o diabetes foi mencionado em 13.786 (6,8\%), sendo a causa básica em 5.305 (2,6\%). A proporção foi maior para mulheres do que para homens (10,1 vs $4,6 \%$ como causa mencionada e 6,1 vs $2,9 \%$ como causa básica). Entre os óbitos com menção de diabetes no atestado, as principais causas básicas foram: diabetes $(38,5 \%)$, doenças cardiovasculares (37,2\%), doenças respiratórias $(8,5 \%)$ e neoplasias $(4,8 \%)$. Quando o diabetes foi a causa básica, as principais causas associadas foram: doenças cardiovasculares $(42,2 \%)$, respiratórias $(10,7 \%)$ e geniturinárias $(10,1 \%)$. Nos casos onde o diabetes figura como causa associada, as principais causas básicas foram as doenças cardiovasculares $(60,5 \%)$, respiratórias $(13,8 \%)$ e neoplásicas $(7,9 \%)$. Apesar das limitações dos dados obtidos dos atestados de óbito, observou-se que o diabetes representa uma importante causa de morte, traduzindo um problema de saúde de grande magnitude. Também, a análise pelas causas múltiplas de morte fornece um perfil da morbidade associada ao diabetes por ocasião do óbito, salientando a importância das doenças cardiovasculares.
\end{abstract}

Diabetes mellitus, mortalidade. Causas múltiplas da morte.

Correspondência para/Correspondence to: Laercio Joel Franco - Rua Botucatu, 740 - 04023-900 - São Paulo, SP - Brasil. 


\begin{abstract}
Introduction
\end{abstract}

Methodology

Results and Conclusions

\section{INTRO DU ÇÃO}

Atualmente, o diabetes mellitus é considerado como uma das principais doenças crônicas que afetam o homem. É um problema de saúde universal, acometendo todas as classes socioeconômicas e afetando populações de países em todos os estágios de desenvolvimento (King e Rewers ${ }^{5}$, 1993).

Sua importância nas últimas décadas vem crescendo em decorrência de vários fatores, como, maiores taxas de urbanização, industrialização, sedentarismo, obesidade, aumento da esperança de vida e à maior sobrevida dos diabéticos. Juntamente com as doenças cardiovasculares, neoplasias malignas e causas externas, o diabetes está entre os quatro mais importantes problemas de saúde, em termos de número de pessoas afetadas, pela considerável morbidade associada à sua presença, pelos custos envolvidos em seu controle e no tratamento de suas complicações, pelas incapacitações que produz e pelo número de anos de vida perdidos, em decorrência da mortalidade prematura.
Nos países em desenvolvimento, particularmente nos recentemente industrializados, existem evidências de que o diabetes está adquirindo características epidêmicas e deverá constituir-se em um dos mais freqüentes e críticos problemas de saúde no século XXI (WHO $\left.{ }^{13}, 1985\right)$.

Levando-se em conta o envelhecimento da população brasileira e o aumento da prevalência do diabetes que vêm ocorrendo nos últimos anos, seria esperado um aumento da participação do diabetes como causa de óbito. Entretanto, a melhoria da assistência à saúde e o aumento da esperança de vida dos diabéticos têm resultado em que esses indivíduos faleçam, não do diabetes propriamente dito, mas sim de suas complicações crônicas, não figurando, portanto, como causa básica de óbito. Este aspecto ressalta a necessidade de se utilizar nos estudos de mortalidade, a análise das causas múltiplas de óbito, quando se deseja conhecer a real importância do diabetes como causa de morte ( Laurenti e col. ${ }^{6}, 1982$; Fuller e col. ${ }^{3}, 1983$; Lessa e col. ${ }^{7}, 1986$; Melo e col. ${ }^{10}$, 1991; Lessa ${ }^{8}$, 1992). 
Assim, o objetivo do presente trabalho é o de avaliar a frequiência das menções do diabetes nas declarações de óbito e as principais causas associadas à sua presença, no Estado de São Paulo, no ano de 1992.

\section{MATERIAL E MÉTO DO}

Os dados sobre a população do Estado de São Paulo e regiões foram obtidos a partir dos Censos Demográficos do IBGE (1970, 1980 e 1991), e das projeções intercensitárias estimadas pela Fundação SEADE. No cálculo de coeficientes padronizados por idade, utilizou-se como padrão, nas comparações entre a Capital e o Interior, a população da Capital estimada para o ano de 1992.

Os indicadores de mortalidade utilizados foram: os coeficientes específicos de mortalidade e a mortalidade proporcional por diabetes. Em algumas situações, foi feita repartição por idades, que corresponde à população com menos e com mais de 40 anos. Devido ao fato do diabetes apresentar maiores prevalências com o progredir da idade, principalmente após a quinta década de vida, seu impacto na mortalidade pode ser melhor observado quando se analisa os coeficientes para a população com mais de 40 anos de idade. Por outro lado, deve ser lembrado que os óbitos por diabetes na população com menos de 40 anos deveriam ser considerados pelos serviços de saúde como "eventos-sentinela", visto que cerca de um terço desses óbitos poderiam ser evitados (Connell e Louden $^{1}, 1983$ e Will e Connell ${ }^{12}$, 1988).

O Sistema de Classificação Automática de Causas de Morte - ACME (Automated Classification of Medical Entities) implantado na Fundação SEADE, permite selecionar a causa básica de óbito através de um algoritmo computadorizado que avalia sistematicamente as causas registradas em cada declaração de óbito. Esse algoritmo incorpora as regras de seleção e modificação da causa básica, a estrutura de códigos da Classificação Internacional de Doenças-CID e as relações etiológicas existentes entre as afecções representadas por esses códigos; permite eliminar algumas falhas e uniformizar os critérios, além de selecionar, automaticamente, a causa básica de morte. Esse sistema ainda apresenta a vantagem de armazenar, para cada registro de óbito, todas as causas mencionadas na declaração, possibilitando a análise da mortalidade por causas múltiplas (Giraldelli e Saad ${ }^{4}, 1984$ ).

\section{RESU LTAD O S E DISC U SSÃO}

\section{Evolução da Mortalidade por Causas no Estado de São Paulo}

Em 1975, o perfil da mortalidade por causas no Estado de São Paulo apontava a predominância das Doenças do Aparelho Circulatório, das Doenças Infecciosas e Parasitárias, do Aparelho Respiratório, dos Neoplasmas e das Causas Externas. Num período de tempo inferior a duas décadas ocorreram modificações neste perfil, provocadas pela acentuada redução da contribuição das Doenças Infecciosas e Parasitárias, pela estabilização do peso das Doenças do Aparelho Respiratório e o aumento relativo do conjunto das Doenças Crônicas Não Transmissíveis e das Causas Externas (Tabela 1).

Entre os grupos de causas cuja importância relativa aumenta no período, destaca-se o das Doenças das Glândulas Endócrinas, da Nutrição e Transtornos Imunológicos que apresentou aumento de $69 \%$. Esse grupo compreende, entre outras doenças, o Diabetes Mellitus, a Síndrome de Deficiência Imunológica Adquirida (AIDS), e as Deficiências Nutricionais. Essas doenças afetam diferentemente a população, segundo a idade, sendo o diabetes mais freqüente entre pessoas maiores de 40 anos, a AIDS entre adultos jovens e as deficiências nutricionais entre crianças.

O perfil de mortalidade de pessoas com 40 ou mais anos de idade, caracterizado pela predominância das doenças crônicas não transmissíveis, se altera no período de 1975-92, no sentido de redução da contribuição relativa das mortes causadas pelas Doenças Infecciosas e Parasitárias (20\%), pelas Doenças do Aparelho Circulatório (15\%) e pelas Causas Externas (9\%), e de aumento relativo das Doenças do Aparelho Respiratório (75\%), do Aparelho Digestivo (38\%), das Glândulas Endócrinas, da Nutrição e do Metabolismo e Transtornos Imunitários (34\%) e dos Neoplasmas (12\%).

Analisando os óbitos para todas as idades, no grupo das Doenças das Glândulas Endócrinas, da Nutrição e do Metabolismo e Transtornos Imunitários, observa-se, entre as mulheres, o predomínio do diabetes, como causa básica de óbito, e entre os homens, dos "Outros Transtornos Metabólicos e Imunitários". Quando analisados os óbitos de pessoas com 40 ou mais anos de idade, o diabetes assume importância ainda maior, representando aproximadamente a metade dos óbitos masculinos desse grupo de causas, e quase $80 \%$ dos óbitos femininos na Capital e no Interior (Tabela 2).

Analisando-se as causas de óbito nas declarações onde o diabetes figurou como causa básica, observase que em aproximadamente três quartos desses óbitos, o diabetes é mencionado sem especificação de complicações (Tabela 3). Obviamente, apenas a presença do diabetes não seria motivo para justificar o óbito.

Apesar dessa restrição, pode-se notar que os óbitos por complicações agudas (cetoacidose e 
Tabela 1- M ortalidade proporcional por alguns grandes grupos de causas, segundo faixa etária. Estado de São Paulo, 1975 a 1992.

\begin{tabular}{|c|c|c|c|c|c|c|c|c|c|c|c|}
\hline \multirow{2}{*}{\multicolumn{2}{|c|}{ Grupo de causas* }} & \multicolumn{5}{|c|}{ Todas as idades } & \multicolumn{5}{|c|}{40 anos e mais } \\
\hline & & 1975 & 1980 & 1985 & 1990 & 1992 & 1975 & 1980 & 1985 & 1990 & 1992 \\
\hline \multicolumn{2}{|c|}{ Números absolutos } & 170,9 & 172,6 & 179,6 & 202,3 & 202,3 & 92,1 & 107,5 & 123,5 & 144,5 & 147,4 \\
\hline & Total & 100,0 & 100,0 & 100,0 & 100,0 & 100,0 & 100,0 & 100,0 & 100,0 & 100,0 & 100,0 \\
\hline I & $\begin{array}{l}\text { D oenças Infecciosas } \\
\text { e Parasitárias }\end{array}$ & 15,2 & 8,3 & 5,0 & 4,0 & 3,7 & 3,6 & 3,0 & 2,9 & 2,9 & 2,9 \\
\hline \multirow{2}{*}{$\begin{array}{l}\text { II } \\
\text { III }\end{array}$} & Neoplasmas & 9,3 & 10,7 & 11,7 & 12,5 & 13,5 & 15,1 & 15,3 & 15,4 & 15,8 & 16,9 \\
\hline & $\begin{array}{l}\text { Doenças das G lând. } \\
\text { Endócr., da N utr. e do } \\
\text { Metab. e Transt. Imun. }\end{array}$ & 3,8 & 4,1 & 4,1 & 5,5 & 6,4 & 4,1 & 4,1 & 4,4 & 5,1 & 5,5 \\
\hline VII & $\begin{array}{l}\text { Doenças do Aparelho } \\
\text { Circulatório }\end{array}$ & 28,7 & 33,4 & 34,3 & 33,0 & 32,5 & 49,4 & 49,9 & 46,7 & 43,5 & 42,0 \\
\hline VIII & $\begin{array}{l}\text { Doenças do Aparelho } \\
\text { Respiratório }\end{array}$ & 10,8 & 10,6 & 10,2 & 11,0 & 10,7 & 6,4 & 7,7 & 9,7 & 11,4 & 11,2 \\
\hline IX & $\begin{array}{l}\text { Doenças do Aparelho } \\
\text { Digestivo }\end{array}$ & 3,1 & 4,3 & 4,8 & 4,7 & 5,1 & 4,2 & 5,3 & 5,6 & 5,3 & 5,8 \\
\hline$x$ & $\begin{array}{l}\text { Doenças do A parelho } \\
\text { Geniturinário }\end{array}$ & 1,5 & 1,5 & 1,6 & 1,6 & 1,6 & 1,9 & 1,8 & 1,9 & 1,9 & 1,9 \\
\hline XVIII & Causas Externas & 8,3 & 9,5 & 12,0 & 12,5 & 12,1 & 5,4 & 5,0 & 5,0 & 5,0 & 4,9 \\
\hline XVI & $\begin{array}{l}\text { Sintomas, Sinais e } \\
\text { Afecções M aldefinidas }\end{array}$ & 8,7 & 5,9 & 5,9 & 6,1 & 6,1 & 8,4 & 6,3 & 6,4 & 6,8 & 6,7 \\
\hline & Demais Causas & 10,6 & 11,8 & 10,3 & 8,9 & 8,4 & 1,6 & 1,7 & 2,0 & 2,2 & 2,2 \\
\hline
\end{tabular}

Fonte: Fundação SEADE

* Grandes Capítulos da Classificação Internacional de Doenças"

Tabela 2- Ó bitos por doenças das glândulas endócrinas, da nutrição e do metabolismo e transtornos imunitários, por sexo e faixa etária. Capital e Interior do Estado de São Paulo,1992.

\begin{tabular}{|c|c|c|c|c|c|c|c|c|}
\hline \multirow{3}{*}{ Grupos de causas* } & \multicolumn{4}{|c|}{ Todas as idades } & \multicolumn{4}{|c|}{40 anos ou mais } \\
\hline & \multicolumn{2}{|c|}{ Capital } & \multicolumn{2}{|c|}{ Interior } & \multicolumn{2}{|c|}{ Capital } & \multicolumn{2}{|c|}{ Interior } \\
\hline & Masc. & Fem. & Masc. & Fem. & Masc. & Fem. & Masc. & Fem. \\
\hline Números absolutos & 2.981 & 1.667 & 4.663 & 3.666 & 1.400 & 1.272 & 2.464 & 2.904 \\
\hline Total & 100,0 & 100,0 & 100,0 & 100,0 & 100,0 & 100,0 & 100,0 & 100,0 \\
\hline Transtornos da glândula & & & & & & & & \\
\hline $\begin{array}{l}\text { Tireóide (240-246). } \\
\text { D oenças de outras glândulas }\end{array}$ & 0,1 & 0,7 & 0,2 & 1,2 & 0,3 & 0,7 & 0,4 & 1,1 \\
\hline Endócrinas (250-259) & 22,6 & 61,2 & 31,0 & 60,6 & 54,5 & 80,5 & 57,9 & 74,6 \\
\hline Diabetes mellitus( 250) & 22,4 & 60,7 & 30,6 & 59,8 & 54,2 & 79,9 & 57,4 & 74,1 \\
\hline Deficiências nutri. (260-267) & 6,1 & 8,5 & 13,5 & 13,0 & 8,4 & 6,2 & 15,0 & 10,6 \\
\hline O utros transt. metabólicos e & & & & & & & & \\
\hline Transt. imun. (270-279) & 71,2 & 29,6 & 55,3 & 25,2 & 36,8 & 12,6 & 26,7 & 13,7 \\
\hline
\end{tabular}

Fonte: Fundação - SEADE

*Classificação Internacional de Doenças" ${ }^{11}$

coma), em sua grande maioria preveníveis, são responsáveis por $14,8 \%$ dos óbitos por diabetes no Estado de São Paulo, com ligeiro excesso no Interior $(16,0 \%)$ em relação à Capital $(12,3 \%)$. Também fica evidente a importante contribuição das complicações circulatórias periféricas nos óbitos por diabetes. Por outro lado, as freqüentes complicações crônicas cerebrovasculares e cardiovasculares do diabetes não aparecem como decorrentes do diabetes, onde elas são a causa básica do óbito, subestimando a importância do diabetes.

Os dados da Tabela 4 apresentam os coeficientes de mortalidade por diabetes observados e padronizados, por 100.000 habitantes, para a Capital,
Interior e Estado de São Paulo para os anos de 1970, 1980, 1990 e 1992.

$\mathrm{Na}$ Capital, verifica-se que os coeficientes observados se mantiveram estáveis, com tendência a decréscimo nos coeficientes padronizados. No Interior, observa-se uma tendência de aumento, tanto nos coeficientes observados como nos padronizados por idade.

\section{Diabetes Como Causa Básica e Associada de Morte}

\section{Coeficientes Específicos}

Analisando-se as informações sobre os coefici- 
entes de mortalidade por diabetes, observa-se que a mortalidade por diabetes no sexo masculino, no grupo etário de 40 ou mais anos, foi maior na Capital do que no Interior, tanto como causa básica ou como causa básica ou associada; no sexo feminino, a mortalidade foi maior na Capital do que no Interior apenas quando se considera o diabetes como causa básica ou associada (Tabela 5).

A mortalidade por diabetes como causa básica, no grupo com menos de 40 anos, é menor na Capital do que no Interior. Provavelmente este fato reflete maiores facilidades de acesso e melhores serviços de saúde na Capital, visto que considerável parcela dos óbitos, nessa faixa etária, decorre de complicações agudas do diabetes que, em sua maioria, são evitáveis ou reversíveis.

No grupo etário com mais de 40 anos, nota-se que a mortalidade por diabetes, como causa básica ou associada, é maior na Capital do que no Interior, sendo 1,5 vezes maior no sexo masculino e 1,1 vezes no feminino. Este fato provavelmente reflete o melhor preenchimento da declaração de óbito na $\mathrm{Ca}$ pital do que no Interior, com maior freqüência de menção do diabetes como causa associada.

Outro aspecto interessante a ser observado nesses dados é quanto à mortalidade por diabetes no grupo etário com 40 ou mais anos, ser sempre maior nas mulheres do que nos homens, tanto na Capital como no Interior. Este fato pode refletir a maior freqüência do conhecimento do diagnóstico da doença entre as mulheres, conforme foi evidenciado por estudo recente, realizado em várias capitais brasileiras, o que favoreceria a menção do diabetes na declaração de óbito (Malerbi e Franco99, 1992).

\section{Mortalidade Proporcional}

A análise da mortalidade proporcional por causas múltiplas confirma a acentuada subestimação da

Tabela 3- Ó bitos com menção de diabetes como causa básica, por tipo de afeç̧ão ou por tipo de complicação segundo sexo. Capital, Interior e Estado de São Paulo, 1992.

\begin{tabular}{|c|c|c|c|c|c|c|c|c|c|}
\hline \multirow[b]{2}{*}{ Causas de óbito* } & \multicolumn{3}{|c|}{ Capital } & \multicolumn{3}{|c|}{ Interior } & \multicolumn{3}{|c|}{ Estado } \\
\hline & Masc. & Fem. & Total & Masc. & Fem. & Total & Masc. & Fem. & Total \\
\hline Números absolutos & 668 & 1.012 & 1.680 & 1.428 & 2.197 & 3.625 & 2.096 & 3.209 & 5.305 \\
\hline Total & 100,0 & 100,0 & 100,0 & 100,0 & 100,0 & 100,0 & 100,0 & 100,0 & 100,0 \\
\hline $\begin{array}{l}\text { Diabetes sem menção (250.0) } \\
\text { de complicação }\end{array}$ & 75,4 & 73,7 & 74,3 & 71,3 & 73,6 & 72,8 & 72,7 & 73,7 & 73,3 \\
\hline Diabetes com cetoacidose (250.1) & 6,1 & 5,0 & 5,5 & 7,6 & 7,6 & 7,6 & 7,1 & 6,8 & 6,9 \\
\hline Diabetes com coma (250.2) & 4,6 & 8,2 & 6,8 & 8,8 & 8,2 & 8,4 & 7,4 & 8,2 & 7,9 \\
\hline $\begin{array}{l}\text { Diabetes com manifestações } \\
\text { renais }(250.3)\end{array}$ & 4,0 & 2,0 & 2,8 & 2,4 & 2,5 & 2,4 & 2,9 & 2,3 & 2,5 \\
\hline $\begin{array}{l}\text { Diabetes com complicações (250.6) } \\
\text { circulátorias periféricas }\end{array}$ & 9,0 & 7,6 & 8,2 & 8,2 & 6,6 & 7,2 & 8,4 & 6,9 & 7,5 \\
\hline $\begin{array}{l}\text { Diabetes com outras manifestações } \\
(250.4,250.5,250.7 \text { e } 250.9)\end{array}$ & 0,9 & 3,5 & 2,4 & 1,7 & 1,5 & 1,6 & 1,5 & 2,1 & 1,9 \\
\hline
\end{tabular}

Fonte : Fundação SEADE

*Classificação Internacional de Doenças ${ }^{\text {II }}$

Tabela 4- Coeficientes de mortalidade por diabetes mellitus segundo o sexo. Capital, Interior e Estado de São Paulo, 1970 1992.

\begin{tabular}{|c|c|c|c|c|c|c|c|c|c|c|c|c|}
\hline \multirow{3}{*}{ Região } & \multirow{2}{*}{\multicolumn{3}{|c|}{1970}} & \multirow{2}{*}{\multicolumn{3}{|c|}{1980}} & \multicolumn{6}{|c|}{ por 100 mil hab. } \\
\hline & & & & & & & & 1990 & & & 1992 & \\
\hline & Masc. & Fem. & Total & Masc. & Fem. & Total & Masc. & Fem. & Total & Masc. & Fem. & Total \\
\hline \multicolumn{13}{|l|}{ Capital } \\
\hline O bservado & 13,1 & 20,9 & 17,1 & 14,2 & 19,6 & 17,0 & 17,2 & 22,2 & 19,8 & 14,3 & 20,1 & 17,3 \\
\hline Padronizado & 16,4 & 29,4 & 22,9 & 17,0 & 24,5 & 20,8 & 17,7 & 23,0 & 20,4 & 14,3 & 20,1 & 17,3 \\
\hline \multicolumn{13}{|l|}{ Interior } \\
\hline O bservado & 7,6 & 13,3 & 10,4 & 10,0 & 16,4 & 13,2 & 12,0 & 19,2 & 15,6 & 12,8 & 19,5 & 16,2 \\
\hline Padronizado & 9,2 & 20,4 & 14,3 & 11,6 & 22,0 & 16,5 & 12,3 & 22,0 & 17,0 & 12,8 & 21,7 & 17,2 \\
\hline \multicolumn{13}{|l|}{ Estado } \\
\hline O bservado & 9,4 & 15,9 & 12,6 & 11,4 & 17,5 & 14,5 & 13,6 & 20,1 & 16,9 & 13,2 & 19,7 & 16,5 \\
\hline Padronizado & 11,3 & 23,4 & 17,0 & 13,3 & 22,9 & 18,0 & 13,9 & 22,3 & 18,1 & 13,2 & 21,2 & 17,2 \\
\hline
\end{tabular}

Fonte: Fundação SEADE

População-padrão: População da Capital em 1992 
importância do diabetes, quando se considera apenas a causa básica de óbito. Na Tabela 6 pode ser observado que a mortalidade proporcional por diabetes, como causa básica, representa apenas 2,7\% do total de óbitos na Capital e 2,6\% no Interior.

Entretanto, quando se considera a mortalidade proporcional, agregando o diabetes como causa básica ou associada, o coeficiente aumenta 3 vezes na Capital $(8,1 \%)$, e 2,4 vezes no Interior $(6,2 \%)$.

O diabetes é mencionado em $10,1 \%$ do total de óbitos femininos (11,5\% na Capital e 9,5\% no Interior), e em $4,6 \%$ do total de óbitos masculinos $(5,8 \%$ na Capital e $4,1 \%$ no Interior).

\section{Diabetes como Causa Básica e Principais Causas Associadas}

Analisando-se as principais causas associadas aos óbitos onde o diabetes figurou como causa básica, nota-se que tanto na Capital como no Interior, em ambos os sexos, as Doenças do Aparelho Circulatório foram as mais freqüentemente associadas. $\mathrm{Na}$ Capital elas foram mencionadas em $44,4 \%$ dos óbitos por diabetes no sexo masculino e em $42,3 \%$ no sexo feminino; no Interior as percentagens foram, respectivamente, 37,0 e 44,4\%. Observa-se que, para o Estado de São Paulo, em 42,2\% dos óbitos, onde o diabetes foi a causa básica, existe associação com doença do aparelho circulatório, refletindo a elevada frequiência de complicações vasculares ateroscleróticas nos diabéticos, por ocasião do óbito (Tabela 7).

\section{Diabetes como Causa Associada - Principais Causas Básicas}

$\mathrm{Na}$ análise dos principais grupos de doenças que figuram como causa básica de óbito, quando o diabetes foi classificado como causa associada, observa-se o grande predomínio das Doenças do Apare-

Tabela 5- Coeficientes de mortalidade por diabetes mellitus, como causa básica e básica ou associada, segundo sexo e idade. Capital, Interior e Estado de São Paulo, 1992.

\begin{tabular}{|c|c|c|c|c|c|c|}
\hline \multirow{4}{*}{ Sexo } & \multirow{2}{*}{\multicolumn{2}{|c|}{ Capital }} & \multicolumn{4}{|c|}{ (por 100 mil hab.) } \\
\hline & & & \multicolumn{2}{|c|}{ Interior } & \multicolumn{2}{|c|}{ Estado } \\
\hline & Básica & Básica & Básica & Básica & Básica & Básica \\
\hline & & ou associada & & ou associada & & ou associada \\
\hline \multicolumn{7}{|l|}{ Masculino } \\
\hline Até 39 anos & 0,8 & 2,0 & 1,3 & 2,1 & 1,2 & 2,0 \\
\hline 40 anos e mais & 54,6 & 181,1 & 48,5 & 119,9 & 50,3 & 138,3 \\
\hline \multicolumn{7}{|l|}{ Feminino } \\
\hline Até 39 anos & 0,9 & 1,5 & 1,0 & 1,6 & 0,9 & 1,5 \\
\hline 40 anos e mais & 69,7 & 203,7 & 73,7 & 178,6 & 72,4 & 186,7 \\
\hline
\end{tabular}

Tabela 6- M ortalidade proporcional por diabetes como causa básica e associada segundo sexo. Capital, Interior e Estado de São Paulo, 1992.

\begin{tabular}{|c|c|c|c|c|c|c|}
\hline \multirow[b]{2}{*}{ Região } & \multicolumn{2}{|c|}{ Total } & \multicolumn{2}{|c|}{ Masculino } & \multicolumn{2}{|c|}{ Feminino } \\
\hline & $\begin{array}{l}\text { Números } \\
\text { Absolutos }\end{array}$ & $\begin{array}{r}\text { Propor } \\
\text { ções }\end{array}$ & $\begin{array}{l}\text { Números } \\
\text { Absolutos }\end{array}$ & $\begin{array}{r}\text { Propor } \\
\text { ções }\end{array}$ & $\begin{array}{l}\text { Números } \\
\text { Absolutos }\end{array}$ & $\begin{array}{r}\text { Propor } \\
\text { ções }\end{array}$ \\
\hline $\begin{array}{l}\text { Capital } \\
\text { Total de óbitos }\end{array}$ & 63.007 & 100,0 & 37.593 & 100,0 & 25.414 & 100,0 \\
\hline Ó bitos com menção de diabetes & 5.099 & 8,1 & 2.188 & 5,8 & 2.911 & 11,5 \\
\hline Como causa básica & 1.680 & 2,7 & 688 & 1,8 & 1.012 & 4,0 \\
\hline Como causa associada & 3.419 & 5,4 & 1.520 & 4,0 & 1.899 & 7,5 \\
\hline $\begin{array}{l}\text { Interior } \\
\text { Total de óbitos }\end{array}$ & 139.134 & 100,0 & 83.664 & 100,0 & 55.470 & 100,0 \\
\hline Ó bitos com menção de diabetes & 8.687 & 6,2 & 3.435 & 4,1 & 5.252 & 9,5 \\
\hline Como causa básica & 3.623 & 2,6 & 1.428 & 1,7 & 2.195 & 4,0 \\
\hline Como causa associada & 5.064 & 3,6 & 2.007 & 2,4 & 3.057 & 5,5 \\
\hline $\begin{array}{l}\text { Estado } \\
\text { Total de óbitos }\end{array}$ & 202.141 & 100,0 & 121.257 & 100,0 & 80.884 & 100,0 \\
\hline Ó bitos com menção de diabetes & 13.786 & 6,8 & 5.623 & 4,6 & 8.163 & 10,1 \\
\hline Como causa básica & 5.303 & 2,6 & 2.096 & 1,7 & 3.207 & 4,0 \\
\hline Como causa associada & 8.483 & 4,2 & 3.527 & 2,9 & 4.956 & 6,1 \\
\hline
\end{tabular}


lho Circulatório, tanto na Capital (56,1\% nos homens e $64,8 \%$ nas mulheres), como no Interior $(56,2 \%$ nos homens e $63,0 \%$ nas mulheres). Estes dados, além de evidenciarem a maior freqüência da menção do diabetes nas mulheres, traduzem a intensa associação que existe entre o diabetes e doenças do aparelho circulatório (Tabela 8).

O segundo grupo mais frequiente como causa básica de óbito, quando o diabetes aparece como causa associada, é o das Doenças do Aparelho Respiratório.
Os Neoplasmas aparecem como terceira causa básica mais frequiente, nos óbitos onde o diabetes é mencionado como causa associada, como mostra a Tabela 9. Dentre os Neoplasmas, o perfil de suas localizações é distinto entre homens e mulheres. Nos homens, o primeiro lugar é ocupado pelas neoplasias de pulmão (20,3\% na Capital e $17,1 \%$ no Interior), enquanto nas mulheres, a localização mais freqüente é a mama (14,5\% na Capital e 14,2\% no Interior).

Em ambos os sexos, porém, destaca-se a elevada

Tabela 7- Freqüência dos grupos de causas associadas aos óbitos por diabetes como causa básica, segundo sexo. Capital, Interior e Estado de São Paulo, 1992.

\begin{tabular}{|c|c|c|c|c|c|c|c|c|c|}
\hline \multirow[b]{2}{*}{ Grupo de causas } & \multicolumn{3}{|c|}{ Capital } & \multicolumn{3}{|c|}{ Interior } & \multicolumn{3}{|c|}{ Estado } \\
\hline & Masc. & Fem. & Total & Masc. & Fem. & Total & Masc. & Fem. & Total \\
\hline Total & 100,0 & 100,0 & 100,0 & 100,0 & 100,0 & 100,0 & 100,0 & 100,0 & 100,0 \\
\hline Doenças do Aparelho Circulatório & 44,4 & 42,3 & 43,1 & 37,0 & 44,4 & 41,5 & 39,5 & 43,8 & 42,2 \\
\hline Sintomas, Sinais, Afec. Maldefinidos & 14,7 & 17,5 & 16,4 & 18,4 & 17,6 & 17,9 & 17,1 & 17,5 & 17,4 \\
\hline Doenças Aparelho Respiratório & 12,9 & 11,0 & 11,7 & 11,3 & 9,5 & 10,2 & 11,8 & 10,0 & 10,7 \\
\hline D oenças A parelho G eniturinário & 10,8 & 10,1 & 10,4 & 10,5 & 9,7 & 10,0 & 10,6 & 9,9 & 10,1 \\
\hline Doenças Gland. End.N utr. e M etabol. & 4,6 & 5,2 & 5,0 & 5,9 & 6,0 & 6,0 & 5,5 & 5,7 & 5,6 \\
\hline D oenças Infecciosas e Parasitárias & 3,7 & 5,7 & 4,9 & 5,2 & 4,5 & 4,8 & 4,7 & 4,9 & 4,8 \\
\hline Doenças do Aparelho Digestivo & 2,5 & 2,0 & 2,2 & 3,5 & 2,7 & 3,0 & 3,2 & 2,4 & 2,7 \\
\hline O utras & 6,4 & 6,2 & 6,3 & 8,2 & 5,6 & 6,6 & 7,6 & 5,8 & 6,5 \\
\hline
\end{tabular}

Tabela 8- Freqüência das causas básicas de óbitos, onde o diabetes figura como causa associada, segundo o sexo. Capital e Interior do Estado de São Paulo, 1992.

\begin{tabular}{|c|c|c|c|c|c|c|c|c|c|}
\hline \multirow[b]{2}{*}{ Causa básica } & \multicolumn{3}{|c|}{ Capital } & \multicolumn{3}{|c|}{ Interior } & \multicolumn{3}{|c|}{ Estado } \\
\hline & Masc. & Fem. & Total & Masc. & Fem. & Total & Masc. & Fem. & Total \\
\hline Total & 100,0 & 100,0 & 100,0 & 100,0 & 100,0 & 100,0 & 100,0 & 100,0 & 100,0 \\
\hline A parelho circulatório & 56,1 & 64,8 & 60,9 & 56,2 & 63,0 & 60,3 & 56,2 & 63,7 & 60,5 \\
\hline Aparelho respiratório & 15,6 & 12,9 & 14,1 & 15,1 & 12,6 & 13,6 & 15,3 & 12,7 & 13,8 \\
\hline Neoplasmas & 9,7 & 8,0 & 8,8 & 7,9 & 6,9 & 7,3 & 8,7 & 7,4 & 7,9 \\
\hline Aparelho digestivo & 6,7 & 5,0 & 5,7 & 7,1 & 5,9 & 6,4 & 6,9 & 5,5 & 6,1 \\
\hline Aparelho geniturinário & 4,1 & 4,3 & 4,2 & 4,6 & 4,7 & 4,7 & 4,4 & 4,5 & 4,5 \\
\hline Demais causas & 7,8 & 5,0 & 6,3 & 9,1 & 6,9 & 7,7 & 8,5 & 6,2 & 7,2 \\
\hline
\end{tabular}

Tabela 9- Freqüência das neoplasias malignas como causa básica de óbito quando o diabetes é causa associada, segundo sexo. Capital, Interior e Estado de São Paulo, 1992.

\begin{tabular}{|c|c|c|c|c|c|c|c|}
\hline \multirow[b]{2}{*}{$\begin{array}{l}\text { Localização } \\
\text { da neoplasia }\end{array}$} & \multicolumn{3}{|c|}{ Masculino } & \multirow[b]{2}{*}{$\begin{array}{l}\text { Localızaçao } \\
\text { da neoplasia }\end{array}$} & \multicolumn{3}{|c|}{ Feminino } \\
\hline & Capital & Interior & Estado & & Capital & Interior & Estado \\
\hline $\mathrm{N} \cong$ de óbitos & 148 & 158 & 306 & $\mathrm{~N} \cong$ de óbitos & 152 & 211 & 363 \\
\hline Total & 100,0 & 100,0 & 100,0 & Total & 100,0 & 100,0 & 100,0 \\
\hline Pulmões & 20,3 & 17,1 & 18,6 & Mamas & 14,5 & 14,2 & 14,3 \\
\hline Próstata & 16,9 & 9,5 & 13,1 & Pâncreas & 9,2 & 9,9 & 9,9 \\
\hline Pâncreas & 8,8 & 11,4 & 10,1 & Cólon & 8,6 & 7,6 & 8,0 \\
\hline Estômago & 6,8 & 8,9 & 7,8 & Pulmões & 7,9 & 7,2 & 7,2 \\
\hline Fígado e vias biliares & 6,8 & 5,7 & 6,2 & Ú tero & 11,8 & 7,1 & 9,1 \\
\hline Sem especificação & 6,1 & 6,3 & 6,2 & Estômago & 7,9 & 6,9 & 6,9 \\
\hline O utras & 34,3 & 41,1 & 38,0 & Sem especificação & 5,9 & 8,3 & 8,3 \\
\hline & & & & O utras & 34,2 & 38,8 & 36,3 \\
\hline
\end{tabular}

Fonte: Fundação SEADE 
proporção de neoplasias de pâncreas, que ocupam a terceira colocação entre os homens ( $8,8 \%$ na Capital e $11,4 \%$ no Interior) e a terceira, entre as mulheres na Capital $(9,2 \%)$ e a terceira no Interior $(9,9 \%)$. O câncer de pâncreas é uma patologia rara na população geral, e a associação entre ele e o diabetes pode sugerir que os portadores dessa neoplasia tem maior probabilidade de apresentar diabetes, provavelmente por fatores locais (infiltração ou destruição do pâncreas, por exemplo). Há evidências recentes, porém, de que o diabetes seja um fator de risco para o câncer de pâncreas (Everhart e Wright ${ }^{2}, 1995$ ).

\section{Diabetes como Causa Básica ou Associada}

A importância da análise por causas múltiplas no estudo da mortalidade por diabetes fica evidente com os dados apresentados na Tabela 10. Do total de atestados de óbito que apresentam menção de diabetes, esta doença foi a causa básica em 32,9\% dos óbitos na Capital e em 41,7\% no Interior. Ou seja, a análise do diabetes como causa básica de óbito representa apenas um terço da sua real contribuição para o total de óbitos, sem levar em conta as limitações da qualidade do preenchimento das declarações de óbito.

Os dados da Tabela 10 evidenciam a presença da morbidade associada ao diabetes, por ocasião da ocorrência do óbito, com destaque para as Doenças do Aparelho Circulatório, que figuram em 40,7\% dos óbitos na Capital e em 35,2\% no Interior.

\section{CONCLUSÕ ES}

Levando em consideração a tendência de aumento da prevalência do diabetes, é de se prever que, numa primeira fase, ocorra uma elevação dos coeficientes de mortalidade por diabetes. Numa segunda fase,

Tabela 10 - Ó bitos com menção de diabetes como causa básica ou associada, segundo sexo. Capital, Interior e Estado de São Paulo, 1992.

\begin{tabular}{|c|c|c|c|c|c|c|c|c|c|}
\hline \multirow[t]{2}{*}{ Causa básica } & \multicolumn{3}{|c|}{ Capital } & \multicolumn{3}{|c|}{ Interior } & \multicolumn{3}{|c|}{ Estado } \\
\hline & Masc. & Fem. & Total & Masc. & Fem. & Total & Masc. & Fem. & Total \\
\hline \multicolumn{10}{|l|}{ Número de óbitos } \\
\hline com menção de diabetes & 2.188 & 2.911 & 5.099 & 3.435 & 5.252 & 8.687 & 5.623 & 8.163 & 13.786 \\
\hline Total & 100,0 & 100,0 & 100,0 & 100,0 & 100,0 & 100,0 & 100,0 & 100,0 & 100,0 \\
\hline Diabetes mellitus & 30,5 & 34,8 & 32,9 & 41,6 & 41,8 & 41,7 & 37,3 & 39,3 & 38,5 \\
\hline Doenças do Ap. Circulatório & 39,0 & 42,0 & 40,7 & 32,9 & 36,7 & 35,2 & 35,2 & 38,6 & 37,2 \\
\hline Doenças do Ap. Respiratório & 10,8 & 8,3 & 9,4 & 8,8 & 7,3 & 7,9 & 9,6 & 7,7 & 8,5 \\
\hline Neoplasmas & 6,8 & 5,2 & 5,9 & 4,6 & 4,0 & 4,2 & 5,3 & 4,4 & 4,8 \\
\hline Doenças do Ap. Digestivo & 4,7 & 3,2 & 3,8 & 4,1 & 3,4 & 3,7 & 4,4 & 3,4 & 3,8 \\
\hline Doenças do Ap. Geniturinário & 2,8 & 2,8 & 2,8 & 2,7 & 2,7 & 2,7 & 2,8 & 2,8 & 2,8 \\
\hline D emais causas & 5,4 & 3,7 & 4,5 & 5,3 & 4,1 & 4,6 & 5,4 & 3,8 & 4,4 \\
\hline
\end{tabular}

Fonte: Fundação SEADE

como decorrência do melhor preenchimento dos atestados de óbito, maior sobrevida dos diabéticos e melhor assistência à saúde, é de se esperar que os diabéticos venham a falecer não pelo diabetes propriamente dito, mas sim pelas suas complicações crônicas. Neste caso pode ocorrer até uma diminuição nos coeficientes de mortalidade por diabetes, pois são suas complicações crônicas que irão figurar como causa básica do óbito. Esse fato ressalta a necessidade de se empregar, nos estudos de mortalidade, a análise por causas múltiplas de óbito, quando se deseja conhecer a real importância do diabetes como causa de morte. Esta abordagem também permite conhecer o perfil da morbidade associada ao diabetes por ocasião do óbito, onde se pode notar a importância da frequência das doenças cardiovasculares. 


\section{REFERÊNCIAS}

1. CONNELL, F.A. \& LOUDEN, J.M. Diabetes mortality in persons under 45 years of age. Am. J. Public Health, 73:1174-7, 1983.

2. EVEHART, J. \& WRIGHT, D. Diabetes mellitus as a risk factor for pancreatic cancer - a meta-analysis. JAMA , 273: 1605-9, 1995

3. FULLER, J.H. et al. Diabetes mortality - new light on an underestimated public health problem. Diabetologia, $\mathbf{2 4}$ $336-41,1983$

4. GIRALDELLI, B.W. \& SAAD, P.M. Considerações sobre a implantação no Estado de São Paulo do Sistema Automático de Classificação de Causas de Morte. Inf. Demogr. São Paulo, (13):137-59, 1984.

5. KING, H. \& REWERS, M. Global estimates for prevalence of diabetes mellitus and impaires glucose tolerance in adults. Diabetes Care, 16:157-77, 1993.

6. LAURENTI, R. et al. Mortalidade por diabetes mellitus no Município de São Paulo (Brasil). Evolução em um período de 70 anos (1900 - 1978) e análise de alguns aspectos sobre associação de causas. Rev. Saúde Pública, 16: 77-91, 1982.

7. LESSA, I. et al. Diabetes mellitus como causa básica e como causa associada de morte em Salvador, Brasil. Arq. Bras.
Med., 60: 467 - 72, 1986.

8. LESSA, I. Tendência da mortalidade proporcional pelo diabetes mellitus nas capitais brasileiras, 1950 - 1985. Bol. Oficina Sanit. Panam., 113: 212-7, 1992.

9. MALERBI, D.A. \& FRANCO, L.J. Multicenter study of the prevalence of diabetes mellitus and impaired glucose tolerance in the urban Brazilian population aged 30-69 year. Diabetes Care, 15:1509-16, 1992.

10. MELO, M.S. et al. Causas múltiplas de morte em diabéticos no Município de Recife, 1987. Rev. Saúde Pública, 25:43542, 1991 .

11. ORGANIZAÇÃO MUNDIAL DA SAÚDE. Classificação Internacional de Doenças, revisão 1975. São Paulo, Centro da OMS para Classificação de Doenças em Português/ Ministério da Saúde/Universidade de São Paulo/Organização Pan-Americana de Saúde, 1978.

12. WILL, J.C. \& CONNELL, F.A. The preventability of "premature mortality": an investigation of early diabetes dealths. Am. J. Public Health, 78: 831-3, 1988.

13. WORLD HEALTH ORGANIZATION. Diabetes mellitus. Geneva, 1985 (WHO-Technical Report Series, 727). 